Research Article

\title{
Global Convergence of Schubert's Method for Solving Sparse Nonlinear Equations
}

\author{
Huiping Cao \\ College of Mathematics and Econometrics, Hunan University, Changsha 410082, China \\ Correspondence should be addressed to Huiping Cao; huiping_cao@hnu.edu.cn
}

Received 7 May 2014; Accepted 28 July 2014; Published 14 October 2014

Academic Editor: Shuangjie Peng

Copyright (C) 2014 Huiping Cao. This is an open access article distributed under the Creative Commons Attribution License, which permits unrestricted use, distribution, and reproduction in any medium, provided the original work is properly cited.

Schubert's method is an extension of Broyden's method for solving sparse nonlinear equations, which can preserve the zero-nonzero structure defined by the sparse Jacobian matrix and can retain many good properties of Broyden's method. In particular, Schubert's method has been proved to be locally and $q$-superlinearly convergent. In this paper, we globalize Schubert's method by using a nonmonotone line search. Under appropriate conditions, we show that the proposed algorithm converges globally and superlinearly. Some preliminary numerical experiments are presented, which demonstrate that our algorithm is effective for large-scale problems.

\section{Introduction}

In this paper, we consider the quasi-Newton method [1] for solving the general nonlinear equation

$$
F(x)=0,
$$

where the function $F: R^{n} \rightarrow R^{n}$ is continuously differentiable. The ordinary quasi-Newton method for solving (1) generates a sequence $\left\{x_{k}\right\}$ by the following iterative scheme:

$$
x_{k+1}=x_{k}+d_{k},
$$

where the quasi-Newton direction $d_{k}$ is obtained by solving the system of linear equations

$$
B_{k} d+F\left(x_{k}\right)=0 .
$$

Here the matrix $B_{k}$ is an approximation to the Jacobian matrix of $F$ at $x_{k}$ which usually satisfies the quasi-Newton condition (i.e., the secant condition):

$$
B_{k+1} s_{k}=y_{k},
$$

where $s_{k}=x_{k+1}-x_{k}$ and $y_{k}=F\left(x_{k+1}\right)-F\left(x_{k}\right)$. The matrix $B_{k}$ can be updated by different quasi-Newton update formulae. However, quasi-Newton method is not applicable for solving large-scale problems due to the density of $B_{k}$. Fortunately, a large-scale problem usually has the property of sparsity, and then it is natural to extend some known quasi-Newton methods based on this property. Early in 1970, Schubert [2] modified Broyden's method and proposed a sparse Broyden's method, that is, the Schubert's method [3] with $B_{k}$ defined in the following way:

$$
B_{k+1}=B_{k}+\sum_{i=1, s(i)_{k} \neq 0}^{n} e_{i} e_{i}^{T} \frac{\left(y_{k}-B_{k} s_{k}\right) s(i)_{k}^{T}}{s(i)_{k}^{T} s(i)_{k}},
$$

where

$$
s(i)_{k}(j)= \begin{cases}0, & \text { if }(i, j) \in E, \\ s_{k}(j), & \text { if }(i, j) \in J,\end{cases}
$$

where $E$ and $J$ are the sparsity patterns of the Jacobian matrix $F^{\prime}(x)$ and $e_{i}$ denotes the $i$ th column of the $n \times n$ identity matrix. It has been proved by Broyden [4] that the Schubert's method is locally convergent when the Jacobian satisfies a Lipschitz condition. Lam [5] further showed the local and superlinear convergence of Schubert's method in the special case when $s(i)_{k} \neq 0, i=1,2, \ldots, n$ at each iteration. As an improvement, Marwil considered the following updated formula:

$$
B_{k+1}=B_{k}+\sum_{i=1}^{n}\left(s(i)_{k}^{T} s(i)_{k}\right)^{+} e_{i}^{T}\left(y_{k}-B_{k} s_{k}\right) e_{i} s(i)_{k}^{T},
$$


where, for a scalar $\alpha \in R$,

$$
\alpha^{+}= \begin{cases}\alpha^{-1}, & \text { if } \alpha \neq 0, \\ 0, & \text { if } \alpha=0 .\end{cases}
$$

Marwil established stronger local and superlinear convergence, which contains the results in [5] as a special case.

Note that the updated formula (7) is not symmetric; therefore, its use is restricted to problems where the symmetry of the updated matrix is not important. The sparse and symmetric quasi-Newton update has attracted much attention [6-8]. Toint [6] and Fletcher [8] previously proposed symmetric updates which met the sparsity and secant conditions simultaneously. Yamashita [9] proposed a new sparse quasi-Newton update, called Matrix Completion QuasiNewton (MCQN) [10], which exploited the sparsity of the Hessian and guaranteed positive definiteness.

So far, most studies in the convergence of sparse quasiNewton methods have focused on their local behaviors. Seldom studies are concerned with the global convergence of those methods. It is a relatively more difficult research topic than optimization. To the author's knowledge, the main work related to the general global convergence of sparse quasiNewton methods is based on the work [10-12]. The purpose of this paper is to study the global and superlinear convergence of the Schubert's method.

The remainder of this paper is organized as follows. In Section 2, we review some properties of Schubert's method. In Section 3, by using a nonmonotone line search [13], we globalize the Schubert's method and prove its global and superlinear convergence under appropriate conditions. In particular, we will show that after finitely many iterations, the unit step length will be accepted. In Section 4, some preliminary numerical results are presented. Finally, we provide some remarks in Section 5.

\section{Schubert's Update}

In this section, we present some useful properties of the Schubert's update. For the sake of convenience, we introduce some notations.

(1) The subspace $Z_{i} \subset R^{n}$ that identifies the sparsity structure of the $i$ th row of Jacobian matrix $F^{\prime}(x)$ is defined as $Z_{i}=\left\{v \in R^{n} \mid v^{T} F^{\prime}(x) e_{i}=0, \forall x \in D\right\} ;$

(2) $Z \subset R^{n} \times R^{n}$ denotes the sparsity structure of the Jacobian matrix $F^{\prime}(x)$ and it is defined as $Z=\{A \in$ $\left.R^{n \times n} \mid A^{T} e_{i} \in Z_{i}, i=1,2, \ldots, n\right\} ;$

(3) the projection operators $S_{i}, i=1,2, \ldots, n$ project vectors onto the subspaces $Z_{i}, i=1,2, \ldots, n$, which particularly makes $S_{i} s_{k}=s(i)_{k}$;

(4) $Q(y, s)=\left\{B \in R^{n \times n} \mid B s=y\right\}$.

Schubert's update (7) is the unique solution to the following minimization problem [3]:

$$
\min \left\{\|\widehat{B}-B\|_{F}: \widehat{B} \in Q(y, s) \cap Z\right\}
$$

Specifically, the following inequality [3] holds for any $J \in Z$, $y, s \in R^{n}$ with $s \neq 0$ :

$$
\begin{aligned}
\left\|B_{k+1}-J\right\|_{F}^{2} & \\
\leq & \left\|B_{k}-J\right\|_{F}^{2}-\frac{\left\|\left(B_{k}-J\right) s_{k}\right\|_{2}^{2}}{\left\|s_{k}\right\|_{2}^{2}} \\
& +\sum_{i=1}^{n}\left(s(i)_{k}^{T} s(i)_{k}\right)^{+}\left(e_{i}^{T}\left(y_{k}-J s_{k}\right)\right)^{2} .
\end{aligned}
$$

The following theorem states the local and superlinear convergence of Schubert's method, which has been proved by Marwil [3].

Theorem 1. Suppose that $F: R^{n} \rightarrow R^{n}$ satisfies the following conditions.

(1) $F$ is continuously differentiable in an open convex set $D_{0}$.

(2) There exists an $x^{*} \in D_{0}$ such that $F\left(x^{*}\right)=0$ and $F^{\prime}\left(x^{*}\right)$ is nonsingular.

(3) There exists $K=\left(k_{1}, k_{2}, \ldots, k_{n}\right)^{T} \in R^{n}$ with $k_{i} \geq 0$ for $i=1,2, \ldots, n$, such that

$$
\left\|e_{i}^{T}\left(F^{\prime}(x)-F^{\prime}(y)\right)\right\|_{2} \leq k_{i}\|x-y\|_{2}, \quad \forall x, y \in D_{0} .
$$

Then there exist constants $\epsilon, \delta>0$ and a nonsingular matrix $B_{0}$ such that if $x_{0} \in D_{0}$ satisfies $\left\|x_{0}-x^{*}\right\|_{2}<\epsilon$ and $\| B_{0}-$ $F^{\prime}\left(x^{*}\right) \|_{F}<\delta$, then

(i) Schubert's method generates $\left\{B_{k}\right\}$ with $B_{k}$ nonsingular for all $k \geq 0$;

(ii) the sequence $\left\{x_{k}\right\}$ converges to $x^{*}$;

(iii) the convergence is superlinear.

It is noticed that the matrix $B_{k+1}$ determined by (7) may be singular even if $B_{k}$ is nonsingular. To overcome such a difficulty, Marwil [3] proposed a nonsingular schubert's method by using

$$
\bar{B}=B+\sum_{i=1}^{n} \theta^{i}\left(s(i)^{T} s(i)\right)^{+} e_{i}^{T}(y-B s) e_{i} s(i)^{T} .
$$

Here, we have omitted the subscript $k$ and used $\bar{B}$ to represent $B_{k+1}$ and $B$ to represent $B_{k}$. The parameters $\theta^{i}$ are chosen so that $\bar{B}$ is nonsingular when $B$ is nonsingular and the details are given below.

Set $C_{0}=B$ and define for $i=1,2, \ldots, n$ as follows:

$$
\begin{aligned}
C_{i} & =C_{0}+\sum_{j=1}^{i} \theta^{j}\left(s(j)^{T} s(j)\right)^{+} e_{j}^{T}(y-B s) e_{j} s(j)^{T} \\
& =C_{i-1}+\theta^{i}\left(s(i)^{T} s(i)\right)^{+} e_{i}^{T}(y-B s) e_{i} s(i)^{T} .
\end{aligned}
$$

Note that $e_{i}^{T} C_{0}=e_{i}^{T} C_{1}=\cdots=e_{i}^{T} C_{i-1}$, and then

$$
C_{i}=C_{i-1}+\theta^{i}\left(s(i)^{T} s(i)\right)^{+} e_{i}^{T}\left(y-C_{i-1} s\right) e_{i} s(i)^{T} .
$$


For a scalar $\alpha \in(0,1), \theta^{i}$ can be chosen to satisfy

$$
\left|\operatorname{det} C_{i}\right| \geq \sqrt[n]{\alpha}\left|\operatorname{det} C_{i-1}\right|, \quad \theta^{i} \in\left[\frac{1-\sqrt[n]{\alpha}}{1+\sqrt[n]{\alpha}}, 1\right] .
$$

Therefore, $|\operatorname{det}(\bar{B})| \geq \alpha|\operatorname{det} B|$, and $\theta^{i}$ can be chosen so that

$$
\bar{B} \text { is nonsigular, } \quad\left|\theta^{i}-1\right| \leq \widehat{\theta}<1 .
$$

The dependence of $\theta^{i}$ on the iteration $k$ is suppressed, but $\widehat{\theta}$ is independent of $k[3]$.

\section{The Algorithm}

In this section, we will globalize Schubert's method. To this end, we introduce a derivative-free line search proposed by Li and Fukushima [13] to determine a step length $\alpha_{k}$.

Algorithm 2. Given constants $\sigma_{1}>0$ and $\beta \in(0,1)$. Let $\alpha_{k}=$ $\beta^{i_{k}}$, where $i_{k}$ is the smallest nonnegative integer such that

$$
\left\|F\left(x_{k}+\beta^{i_{k}} d_{k}\right)\right\| \leq\left\|F\left(x_{k}\right)\right\|-\sigma_{1}\left\|\beta^{i_{k}} d_{k}\right\|^{2}+\varepsilon_{k}\left\|F\left(x_{k}\right)\right\|,
$$

and $\left\{\varepsilon_{k}\right\}$ is a given positive sequence satisfying

$$
\sum_{k=0}^{\infty} \varepsilon_{k} \leq \varepsilon<\infty
$$

It is not difficult to see that Algorithm 2 is well defined. Moreover, for each $k$, we have

$$
\left\|F\left(x_{k}+\alpha_{k} d_{k}\right)\right\| \leq\left(1+\varepsilon_{k}\right)\left\|F\left(x_{k}\right)\right\| .
$$

By using Algorithm 2, we give our algorithm as follows.

Algorithm 3. Consider the following.

Step 0. Given constants $\rho, \beta, \bar{\theta} \in(0,1), \sigma_{1}, \sigma_{2}>0$, select a positive sequence $\left\{\varepsilon_{k}\right\}$ satisfying (18). Then choose an initial point $x_{0} \in R^{n}$ and a nonsingular matrix $B_{0} \in R^{n \times n}$. Let $k:=0$.

Step 1. Stop if $F\left(x_{k}\right)=0$. Otherwise, solve the following system of linear equations

$$
B_{k} d+F\left(x_{k}\right)=0
$$

to get $d_{k}$.

Step 2. If

$$
\left\|F\left(x_{k}+d_{k}\right)\right\| \leq \rho\left\|F\left(x_{k}\right)\right\|-\sigma_{2}\left\|d_{k}\right\|^{2},
$$

then let $\alpha_{k}:=1$ and go to Step 4 . Else, go to Step 3 .

Step 3. Let $\alpha_{k}$ be determined by Algorithm 2 .

Step 4. Set $x_{k+1}:=x_{k}+\alpha_{k} d_{k}$.

Step 5. Compute $B_{k+1}$ by (12).

Step 6. Set $k:=k+1$. Go to Step 1 .

We then show some useful properties of Algorithm 3.
Lemma 4. Let the level set $\Omega=\left\{x \in R^{n} \mid\|F(x)\| \leq e^{\varepsilon}\left\|F\left(x_{0}\right)\right\|\right\}$ be bounded and let $\left\{x_{k}\right\}$ be generated by Algorithm 3. Then $\left\{x_{k}\right\}$ is contained in $\Omega$. Moreover, it holds that

$$
\sum_{k=0}^{\infty}\left\|s_{k}\right\|^{2}<\infty
$$

Proof. By the line search (17), we have for any $k$

$$
\left\|F\left(x_{k+1}\right)\right\| \leq\left(1+\varepsilon_{k}\right)\left\|F\left(x_{k}\right)\right\|
$$

$$
\begin{aligned}
& \leq\left\|F\left(x_{0}\right)\right\|\left[\prod_{j=0}^{k}\left(1+\varepsilon_{j}\right)\right] \\
& \leq\left\|F\left(x_{0}\right)\right\|\left[\frac{1}{k+1} \sum_{j=0}^{k}\left(1+\varepsilon_{j}\right)\right]^{k+1} \\
& =\left\|F\left(x_{0}\right)\right\|\left[1+\frac{1}{k+1} \sum_{j=0}^{k} \varepsilon_{j}\right]^{k+1} \\
& \leq\left\|F\left(x_{0}\right)\right\|\left[\left(1+\frac{\varepsilon}{k+1}\right)^{(k+1) / \varepsilon}\right]^{\varepsilon} \\
& \leq e^{\varepsilon}\left\|F\left(x_{0}\right)\right\| .
\end{aligned}
$$

This implies that the sequence $\left\{x_{k}\right\}$ generated by Algorithm 3 is contained in $\Omega$ and the sequence $\left\{\left\|F\left(x_{k}\right)\right\|\right\}$ is bounded. Moreover, combined with (17) and (21), we can get for each $k$

$$
\begin{aligned}
\sigma_{0}\left\|s_{k}\right\|^{2} & =\sigma_{0}\left\|x_{k+1}-x_{k}\right\|^{2} \\
& \leq\left\|F\left(x_{k}\right)\right\|-\left\|F\left(x_{k+1}\right)\right\|+\varepsilon_{k}\left\|F_{k}\right\|,
\end{aligned}
$$

where $\sigma_{0}=\min \left\{\sigma_{1}, \sigma_{2}\right\}$. Making summation on both sides for $k$ from 0 to $\infty$, we obtain (22).

Similar to Lemma 2.4 in [13], we have the following result.

Lemma 5. Let the level set $\Omega$ be bounded and let $\left\{x_{k}\right\}$ be generated by Algorithm 3. Then the sequence $\left\{\left\|F\left(x_{k}\right)\right\|\right\}$ is convergent.

In order to establish the global convergence of Algorithm 3, we need the following assumptions.

Assumption 6. (i) The level set $\Omega$ defined in Lemma 4 is bounded.

(ii) $F(x)$ is continuously differentiable in an open set $D \subseteq$ $\Omega$, and there exists an $x^{*} \in \Omega$ such that $F\left(x^{*}\right)=0$ and $F^{\prime}\left(x^{*}\right)$ is nonsingular.

(iii) $F^{\prime}(x)$ is Lipschitz continuous on $\Omega$; that is, there exists an $L>0$ such that

$$
\left\|F^{\prime}(x)-F^{\prime}(y)\right\| \leq L\|x-y\|, \quad \forall x, y \in \Omega .
$$

(iv) $F^{\prime}(x)$ is nonsingular for any $x \in \Omega$. 
Assumption 6 (iv) is the same as that in [13], which is not as strong as the assumption adopted in [14], where the uniform nonsingularity of $F^{\prime}(x)$ is assumed.

We first introduce some notations. Define

$$
A_{k+1}=\int_{0}^{1} F^{\prime}\left(x_{k}+t s_{k}\right) d t,
$$

and then we have $y_{k}=A_{k+1} s_{k}$. Let

$$
\begin{aligned}
\eta_{k} & =\left\|\sum_{i=1}^{n}\left(s(i)_{k}^{T} s(i)_{k}\right)^{+} e_{i}^{T}\left(y_{k}-B_{k} s_{k}\right) e_{i} s(i)_{k}^{T}\right\|, \\
\delta_{k} & =\frac{\left\|y_{k}-B_{k} s_{k}\right\|}{\left\|s_{k}\right\|}=\frac{\left\|\left(A_{k+1}-B_{k}\right) s_{k}\right\|}{\left\|s_{k}\right\|} \\
& =\frac{1}{\left\|s_{k}\right\|}\left\|\sum_{i=1}^{n}\left(s(i)_{k}^{T} s(i)_{k}\right)^{+} e_{i}^{T}\left(y_{k}-B_{k} s_{k}\right) e_{i} s(i)_{k}^{T} s_{k}\right\| .
\end{aligned}
$$

The following lemma is an extension of Lemma 2.5 in [13].

Lemma 7. Let the sequence $\left\{x_{k}\right\}$ be generated by Algorithm 3 . Suppose that the conditions (i)-(iii) in Assumption 6 hold. If

$$
\sum_{k=0}^{\infty}\left\|s_{k}\right\|^{2}<\infty
$$

then one has

$$
\lim _{l \rightarrow \infty} \frac{1}{l} \sum_{k=0}^{l-1} \delta_{k}^{2}=0 .
$$

In particular, there is a subsequence of $\left\{\delta_{k}\right\}$ tending to zero. If

$$
\sum_{k=0}^{\infty}\left\|s_{k}\right\|<\infty
$$

then one has

$$
\sum_{k=0}^{\infty} \delta_{k}^{2}<\infty
$$

In particular, the whole sequence $\left\{\delta_{k}\right\}$ converges to zero.

Proof. By the Lipschitz continuity of $F^{\prime}$, we have

$$
\left\|A_{k+1}-A_{k}\right\| \leq \frac{1}{2} L\left(\left\|s_{k}\right\|+\left\|s_{k-1}\right\|\right) .
$$

Denote

$$
a_{k}=\left\|B_{k}-A_{k}\right\|_{F}, \quad b_{k}=\left\|A_{k+1}-A_{k}\right\|_{F} .
$$

According to the updated (12), we have

$$
e_{i}^{T} B_{k+1}=e_{i}^{T} B_{k}+\left(s(i)_{k}^{T} s(i)_{k}\right)^{+} \theta_{k}^{i} e_{i}^{T}\left(y_{k}-B_{k} s_{k}\right) s(i)_{k}^{T} .
$$

Subtracting $e_{i}^{T} A_{k+1}$ from both sides of the above equality gives

$$
\begin{aligned}
e_{i}^{T}\left(B_{k+1}-A_{k+1}\right) & \\
\quad= & e_{i}^{T}\left(B_{k}-A_{k+1}\right)+\theta_{k}^{i} e_{i}^{T}\left(s(i)_{k}^{T} s(i)_{k}\right)^{+}\left(y_{k}-B_{k} s_{k}\right) s(i)_{k}^{T} \\
& =e_{i}^{T}\left(B_{k}-A_{k+1}\right)\left(I-\theta_{k}^{i}\left(s(i)_{k}^{T} s(i)_{k}\right)^{+} s(i)_{k} s(i)_{k}^{T}\right) .
\end{aligned}
$$

Taking norms yields

$$
\begin{aligned}
&\left\|e_{i}^{T}\left(B_{k+1}-A_{k+1}\right)\right\|^{2} \\
&=\left\|e_{i}^{T}\left(B_{k}-A_{k+1}\right)\left(I-\theta_{k}^{i}\left(s(i)_{k}^{T} s(i)_{k}\right)^{+} s(i)_{k} s(i)_{k}^{T}\right)\right\|^{2} \\
&=\left\|e_{i}^{T}\left(B_{k}-A_{k+1}\right)\right\|^{2} \\
&-\theta_{k}^{i}\left(2-\theta_{k}^{i}\right)\left(s(i)_{k}^{T} s(i)_{k}\right)^{+}\left\|e_{i}^{T}\left(B_{k}-A_{k+1}\right) s(i)_{k} s(i)_{k}^{T}\right\|^{2} .
\end{aligned}
$$

Making summation on both sides, $i=1, \ldots, n$, yields

$$
\begin{aligned}
\| B_{k+1}- & A_{k+1}\left\|_{F}^{2}=\sum_{i=1}^{n}\right\| e_{i}^{T}\left(B_{k+1}-A_{k+1}\right) \|^{2} \\
= & \sum_{i=1}^{n}\left\|e_{i}^{T}\left(B_{k}-A_{k+1}\right)\right\|^{2} \\
& -\sum_{i=1}^{n} \theta_{k}^{i}\left(2-\theta_{k}^{i}\right)\left(s(i)_{k}^{T} s(i)_{k}\right)^{+} \\
& \times\left\|e_{i}^{T}\left(B_{k}-A_{k+1}\right) s(i)_{k} s(i)_{k}^{T}\right\|^{2} \\
= & \left\|\left(B_{k}-A_{k+1}\right)\right\|_{F}^{2} \\
& -\| \sum_{i=1}^{n} \theta_{k}^{i}\left(2-\theta_{k}^{i}\right) e_{i} e_{i}^{T}\left(s(i)_{k}^{T} s(i)_{k}\right)^{+} \\
& \times\left(y_{k}-B_{k} s_{k}\right) s(i)_{k}^{T} \|^{2} \\
\leq & \left\|\left(B_{k}-A_{k+1}\right)\right\|_{F}^{2}-\left(1-\hat{\theta}^{2}\right) \\
& \times\left\|\sum_{i=1}^{n} e_{i} e_{i}^{T}\left(s(i)_{k}^{T} s(i)_{k}\right)^{+}\left(y_{k}-B_{k} s_{k}\right) s(i)_{k}^{T}\right\|^{2} \\
= & \left\|\left(B_{k}-A_{k+1}\right)\right\|_{F}^{2}-\left(1-\hat{\theta}^{2}\right) \eta_{k}^{2} .
\end{aligned}
$$

Then it follows that

$$
\begin{aligned}
\left(1-\widehat{\theta}^{2}\right) \eta_{k}^{2} & \leq\left\|B_{k}-A_{k+1}\right\|_{F}^{2}-a_{k+1}^{2} \\
& \leq\left(a_{k}+b_{k}\right)^{2}-a_{k+1}^{2} \\
& =a_{k}^{2}-a_{k+1}^{2}+2 a_{k} b_{k}+b_{k}^{2}, \\
a_{k+1}^{2} & \leq\left(a_{k}+b_{k}\right)^{2}-\left(1-\widehat{\theta}^{2}\right) \eta_{k}^{2} .
\end{aligned}
$$

According to Lemma 2.5 of [13], we get

$$
\begin{gathered}
\lim _{l \rightarrow \infty} \frac{1}{l} \sum_{k=0}^{l-1} \eta_{k}^{2}=0, \\
\sum_{k=0}^{\infty} \eta_{k}^{2}<\infty .
\end{gathered}
$$


Moreover, for each $k$ we have

$$
\begin{aligned}
\delta_{k} & =\frac{1}{\left\|s_{k}\right\|}\left\|\sum_{i=1}^{n}\left(s(i)_{k}^{T} s(i)_{k}\right)^{+} e_{i}^{T}\left(y_{k}-B_{k} s_{k}\right) e_{i} s(i)_{k}^{T} s_{k}\right\| \\
& \leq\left\|\sum_{i=1}^{n}\left(s(i)_{k}^{T} s(i)_{k}\right)^{+} e_{i}^{T}\left(y_{k}-B_{k} s_{k}\right) e_{i} s(i)_{k}^{T}\right\|=\eta_{k} .
\end{aligned}
$$

This completes the proof.

According to Algorithm 3, we have the following lemma.

Lemma 8. Let $\left\{x_{k}\right\}$ be generated by Algorithm 3. If there are finitely many $k$ for which $\alpha_{k}$ is determined by (21), then one has

$$
\lim _{t \rightarrow \infty} \frac{1}{t} \sum_{k=0}^{t} \sum_{i=1}^{m} \delta_{k}^{2}=0
$$

In particular, there is an infinity index set $K$ such that, for each $i=1,2, \ldots, m$, the subsequence $\left\{\delta_{k}\right\}_{k \in K}$ converges to zero.

Proof. Since there are finitely many $k$ for which $\alpha_{k}$ is determined by (21), we can know that there exists an index $\widehat{k}$ such that, for $k>\widehat{k}$,

$$
\left\|F\left(x_{k}+\rho^{i} d_{k}\right)\right\| \leq\left\|F\left(x_{k}\right)\right\|-\sigma_{1}\left\|\rho^{i} d_{k}\right\|^{2}+\epsilon_{k}\left\|F\left(x_{k}\right)\right\| .
$$

This implies

$$
\sum_{k=\widehat{k}}^{\infty}\left\|s_{k}\right\|^{2}=\sum_{k=\widehat{k}}^{\infty}\left\|x_{k+1}-x_{k}\right\|^{2}=\sum_{k=\widehat{k}}^{\infty}\left\|\alpha_{k} d_{k}\right\|^{2}<\infty .
$$

According to Lemma 7, we can easily prove the result.

Lemma 9. Suppose that $\sum_{k=1}^{\infty}\left\|s_{k}\right\|^{2}<\infty$ and that there is an accumulation point $x^{*}$ of $\left\{x_{k}\right\}_{k \in K}$ at which $F^{\prime}\left(x^{*}\right)$ is nonsingular. Then there exists a constant $M_{1}>0$ such that the following inequality holds for all $k \in K$ sufficiently large:

$$
\left\|d_{k}\right\| \leq M_{1}\left\|F\left(x_{k}\right)\right\| \text {. }
$$

Proof. Without loss of generality, we suppose $\left\{x_{k}\right\}_{K} \rightarrow x^{*}$. Since $\sum_{k=1}^{\infty}\left\|s_{k}\right\|^{2}<\infty$, it is clear that when $k \in K$ is sufficiently large, $A_{k+1}$ is nonsingular. Moreover, there is a constant $C_{1}>0$ such that the inequality $\left\|A_{k+1}^{-1}\right\| \leq C_{1}$ holds for all $k \in K$ sufficiently large. It then follows from Lemma 8 that $\left\{\delta_{k}\right\}_{K} \rightarrow 0$. Therefore, there exists an index $N>0$ such that the inequality $\delta_{k} \leq 1 /\left(2 C_{1}\right)$ holds for all $k \in K$ with $k \geq$ $N$. Consequently, we get from the definition of $\delta_{k}$ that for all $k \in K$ with $k>N$ sufficiently large

$$
\begin{aligned}
\left\|d_{k}\right\| & \leq\left\|A_{k+1}^{-1}\right\|\left(\left\|A_{k+1} d_{k}+g\left(x_{k}\right)\right\|+\left\|g\left(x_{k}\right)\right\|\right) \\
& \leq C_{1}\left(\left\|g\left(x_{k}\right)\right\|+\delta_{k}\left\|d_{k}\right\|\right) \leq C_{1}\left(\left\|g\left(x_{k}\right)\right\|+\frac{1}{2 C_{1}}\left\|d_{k}\right\|\right) \\
& \leq C_{1}\left\|g\left(x_{k}\right)\right\|+\frac{1}{2}\left\|d_{k}\right\| .
\end{aligned}
$$

The last inequality implies (44) with $M_{1}=2 C_{1}$.
We show the global convergence of Algorithm 3 in the following section.

Theorem 10. Let Assumption 6 hold and let index set $K$ be specified by Lemma 8 . Then the sequence $\left\{x_{k}\right\}$ converges to the unique solution $x^{*}$ of (1).

Proof. We first verify

$$
\lim _{k \rightarrow \infty} \inf \left\|F\left(x_{k}\right)\right\|=0 .
$$

If there are infinitely many $k$ for which $\alpha_{k}$ is determined by (21), then $\left\|F\left(x_{k+1}\right)\right\| \leq \rho\left\|F\left(x_{k}\right)\right\|$ holds for infinitely many $k$. Let $I$ be the index set for which (21) holds and let $i_{k}$ be the number of index $j$, where $j \leq k$ and $j \in I$. Then we can know that $i_{k} \rightarrow \infty$, when $k \rightarrow \infty$. For each $k \notin I$, we have $\left\|F\left(x_{k+1}\right)\right\| \leq\left(1+\epsilon_{k}\right)\left\|F\left(x_{k}\right)\right\|$, and then for all sufficiently large $k$ we have

$$
\left\|F\left(x_{k+1}\right)\right\| \leq(\rho)^{i_{k+1}} \prod_{i=0}^{k}\left(1+\epsilon_{i}\right)\left\|F\left(x_{0}\right)\right\| \leq \rho^{i_{k+1}} e^{\epsilon}\left\|F\left(x_{0}\right)\right\|,
$$

where $\rho \in(0,1)$. This implies $\liminf _{k \rightarrow \infty}\left\|F\left(x_{k}\right)\right\|=0$, and hence the conclusion is true.

If there are finitely many $k$ for which $\alpha_{k}$ is determined by (21), by Lemma 8 , there exists a subsequence $\left\{\delta_{k}\right\}_{k \in K}$ that converges to zero. Similar to the proof of Lemma 9, it is not difficult to show that (44) holds for all $k \in \bar{K}$ sufficiently large, where $\bar{K}$ denotes the index set of $k>\widehat{k}, k \in K$. In particular, the subsequence $\left\{d_{k}\right\}_{\bar{K}}$ is bounded. Without loss of generality, we suppose that $\left\{d_{k}\right\}_{\bar{K}}$ converges to some $d^{*}$.

Denote $\bar{\alpha}=\limsup _{k \rightarrow \infty} \alpha_{k}$. It is clear that $\bar{\alpha} \geq 0$. If $\bar{\alpha}>0$, then $d^{*}=0$, and hence it follows from (20) that $F\left(x^{*}\right)=0$. Suppose $\bar{\alpha}=0$, or equivalently $\lim _{k \rightarrow \infty} \alpha_{k}=0$. By the line search rule, when $k \in \bar{K}$ is sufficiently large, $\alpha_{k}<1$ and hence

$$
\left\|F\left(x_{k}+\rho^{-1} \alpha_{k} d_{k}\right)\right\|-\left\|F\left(x_{k}\right)\right\|>-\sigma_{1}\left\|\rho^{-1} \alpha_{k} d_{k}\right\|^{2} .
$$

Multiplying both sides by $\left(\left\|F\left(x_{k}+\rho^{-1} \alpha_{k} d_{k}\right)\right\|+\right.$ $\left.\left\|F\left(x_{k}\right)\right\|\right) /\left(\rho^{-1} \alpha_{k}\right)$ and then taking limit as $k \rightarrow \infty$ with $k \in \bar{K}$, we obtain

$$
F\left(x^{*}\right)^{T} F^{\prime}\left(x^{*}\right) d^{*} \geq 0 .
$$

On the other hand, taking the limit in (20) as $k \rightarrow \infty$ with $k \in \bar{K}$ yields

$$
F^{\prime}\left(x^{*}\right) d^{*}+F\left(x^{*}\right)=0
$$

This together with (49) implies $F\left(x^{*}\right)=0$.

In the latter part of this section, we give the superlinear convergence of Algorithm 3.

Theorem 11. Let the conditions in Theorem 10 hold. Then there exist a constant $\delta>0$ and an index $\bar{k}$ such that $\alpha_{k}=1$ whenever $\delta_{k} \leq \delta$ and $k \geq \bar{k}$, the inequality

$$
\left\|F\left(x_{k}+d_{k}\right)\right\| \leq \rho\left\|F\left(x_{k}\right)\right\|-\sigma_{1}\left\|d_{k}\right\|^{2}<\rho\left\|F\left(x_{k}\right)\right\|
$$

holds for all $k \geq \bar{k}$ and $\delta_{k} \leq \delta$. 
Proof. By Theorem 10, $\left\{x_{k}\right\}$ converges to the solution of (1), say, $x^{*}$, and there exists a constant $C_{2}>0$ such that $\left\|A_{k+1}^{-1}\right\| \leq$ $C_{2}$ for all $k$ sufficiently large. Similar to the proof of Lemma 9 , when $k$ is large enough we can show that

$$
\left\|d_{k}\right\| \leq M_{2}\left\|F\left(x_{k}\right)\right\|
$$

And from (20) we have

$$
\begin{aligned}
A_{k+1} & \left(x_{k}+d_{k}-x^{*}\right) \\
& =A_{k+1}\left(x_{k}-x^{*}\right)+\left(A_{k+1}-B_{k}\right) d_{k}-F\left(x_{k}\right) \\
& =\left(A_{k+1}-F^{\prime}\left(x^{*}\right)\right)\left(x_{k}-x^{*}\right)+\left(A_{k+1}-B_{k}\right) d_{k} \\
& -F\left(x_{k}\right)+F\left(x^{*}\right)+F^{\prime}\left(x^{*}\right)\left(x_{k}-x^{*}\right),
\end{aligned}
$$

and this implies

$$
\begin{aligned}
\left\|x_{k}+d_{k}-x^{*}\right\| \leq & \left\|A_{k+1}^{-1}\right\| \\
& \times\left(\left\|A_{k+1}-F^{\prime}\left(x^{*}\right)\right\|\right. \\
& \times\left\|x_{k}-x^{*}\right\|+\left\|\left(A_{k+1}-B_{k}\right) d_{k}\right\| \\
& +\| F\left(x_{k}\right)-F\left(x^{*}\right) \\
& \left.+F^{\prime}\left(x^{*}\right)\left(x_{k}-x^{*}\right) \|\right) \\
\leq & C_{2}\left(o\left\|x_{k}-x^{*}\right\|^{2}+\delta_{k}\left\|d_{k}\right\|\right) \\
\leq & C_{2}\left(o\left\|x_{k}-x^{*}\right\|\right. \\
& \left.+M_{2} \delta_{k}\left\|F\left(x_{k}\right)-F\left(x^{*}\right)\right\|\right) \\
\leq & C_{2}\left(o\left\|x_{k}-x^{*}\right\|+M_{2} M \delta_{k}\left\|x_{k}-x^{*}\right\|\right),
\end{aligned}
$$

where $M$ is an upper bound of $F^{\prime}(x)$ in $\Omega$. The second and third inequalities follow from the definition of $\delta_{k}$ and (52), respectively. It then follows that

$$
\begin{aligned}
\left\|F\left(x_{k}+d_{k}\right)\right\| & =\left\|F\left(x_{k}+d_{k}\right)-F\left(x^{*}\right)\right\| \\
& \leq M\left\|x_{k}+d_{k}-x^{*}\right\| \\
& \leq M C_{2}\left(o\left\|x_{k}-x^{*}\right\|+M_{2} M \delta_{k}\left\|x_{k}-x^{*}\right\|\right) .
\end{aligned}
$$

On the other hand, by the nonsingularity of $F^{\prime}\left(x^{*}\right)$ and the fact that $x_{k} \rightarrow x^{*}$, there is a constant $m>0$ such that

$$
\left\|F\left(x_{k}\right)\right\|=\left\|F\left(x_{k}\right)-F\left(x^{*}\right)\right\| \geq m\left\|x_{k}-x^{*}\right\|
$$

holds for all $k$ sufficiently large. Therefore, we deduce from (52) and (56) that when $\delta_{k} \leq \delta^{\prime}$

$$
\begin{aligned}
&\left\|F\left(x_{k+1}\right)\right\|-\rho\left\|F\left(x_{k}\right)\right\|+\sigma_{2}\left\|d_{k}\right\|^{2} \\
& \leq M C_{2}\left(o\left\|x_{k}-x^{*}\right\|+M_{2} M \delta_{k}\left\|x_{k}-x^{*}\right\|\right) \\
&-\rho m\left\|x_{k}-x^{*}\right\|+\sigma_{2} M_{2}^{2}\left\|F\left(x_{k}\right)\right\|^{2} \\
& \leq\left(M_{2} C_{2} M^{2} \delta_{k}-\rho m\right)\left\|x_{k}-x^{*}\right\| \\
&+o\left(\left\|x_{k}-x^{*}\right\|\right)+\sigma_{2} M_{2}^{2} M^{2}\left\|x_{k}-x^{*}\right\|^{2} \\
& \leq-\left(\rho m-M_{2} C_{2} M^{2} \delta_{k}\right)\left\|x_{k}-x^{*}\right\| \\
&+o\left(\left\|x_{k}-x^{*}\right\|\right) .
\end{aligned}
$$

Let $\delta=\min \left\{\delta^{\prime},(1 / 2) \rho m\left(M_{2} C_{2} M^{2}\right)^{-1}\right\}$. Then we know that when $\delta_{k}<\delta$, (51) holds for all $k$ sufficient large.

The following theorem establishes the superlinear convergence of Algorithm 3.

Theorem 12. Let the conditions in Theorem 10 hold. Then the sequence $\left\{x_{k}\right\}$ generated by Algorithm 3 converges to the unique solution $x^{*}$ of (1) superlinearly.

Proof. By Theorem 11, it suffices to show $\left\{\delta_{k}\right\} \rightarrow 0$ as $k \rightarrow$ $\infty$.

Let $\delta$ and $\bar{k}$ be as specified by Theorem 11. It follows from Lemma 8 that there is an index $\widetilde{k}$ such that the following inequality holds for all $k \geq \widetilde{k}$ :

$$
\frac{1}{k} \sum_{j=0}^{k-1} \delta_{j}^{2} \leq \frac{1}{2} \delta^{2}
$$

This shows that, for any $k \geq \widetilde{k}$, there are at least $\lceil k / 2\rceil$ many indices $j \leq k$ such that $\delta_{j} \leq \delta$. Let $k^{\prime}=\max \{\bar{k}, \widetilde{k}\}$. By Theorem 11 , for any $k \geq 2 k^{\prime}$, there are at least $\lceil k / 2\rceil-k^{\prime}$ many indices $j \leq k$ such that $\alpha_{j}=1$ and

$$
\left\|F\left(x_{j+1}\right)\right\|=\left\|F\left(x_{j}+d_{k}\right)\right\| \leq \rho\left\|F\left(x_{j}\right)\right\| .
$$

Let $J_{k}$ be the set of indices for which (21) holds and let $j_{k}$ be the number of elements in $J_{k}$. Then $j_{k} \geq(k / 2)-k^{\prime}-1$. On the other hand, for each $j \notin J_{k}$, we have

$$
\left\|F\left(x_{j+1}\right)\right\| \leq\left(1+\varepsilon_{k}\right)\left\|F\left(x_{j}\right)\right\| .
$$

Multiplying inequalities (21) with $j \in J_{k}$ and (60) with $j \notin J_{k}$, we can obtain for any $k>2 k^{\prime}$

$$
\begin{aligned}
\left\|F\left(x_{k+1}\right)\right\| & \leq(\rho)^{j_{k}}\left\|F\left(x_{k^{\prime}}\right)\right\|\left[\prod_{j=k^{\prime}}^{k}\left(1+\varepsilon_{j}\right)\right] \\
& \leq\left\|F\left(x_{k^{\prime}}\right)\right\| \rho^{(1 / 2) k-k^{\prime}-1} e^{\varepsilon},
\end{aligned}
$$


or equivalently

$$
\left\|F\left(x_{k+1}\right)\right\| \leq\left\|F\left(x_{k^{\prime}}\right)\right\| \rho^{(1 / 2) k-k^{\prime}-1} e^{\varepsilon} .
$$

So, we have

$$
\sum_{k=0}^{\infty}\left\|F\left(x_{k}\right)\right\|<\infty .
$$

This together with (56) implies

$$
\sum_{k=0}^{\infty}\left\|s_{k}\right\|<\infty .
$$

It then follows from Lemma 7 that $\left\{\delta_{k}\right\} \rightarrow 0$ as $k \rightarrow \infty$. The proof is completed.

\section{Numerical Experiments}

In this section, we will present some numerical results to show the efficiency of Algorithm 3 for a class of sparse nonlinear equations.

In each experiment, we employ the following termination criterion:

$$
\left\|F\left(x_{k}\right)\right\|_{2} \leq 10^{-5}
$$

The parameters in Algorithm 3 are specified as follows:

$$
\begin{gathered}
\rho=0.9, \quad \sigma_{1}=\sigma_{2}=0.001, \quad \beta=0.45, \\
\eta_{k}=\frac{1}{(k+1)^{2}},
\end{gathered}
$$

see [13].

The numerical experiments are done by using MATLAB version 7.10 on a Core (TM) 2 PC with Windows XP. The details of the problems are given as follows, where $x_{0}$ denotes the initial point.

Problem 1 (Broyden tridiagonal function [15]). The elements of $F(x)$ are

$$
\begin{gathered}
F_{1}(x)=-\left(3-0.5 x_{1}\right) x_{1}+2 x_{2}-1, \\
F_{i}(x)=x_{i-1}-\left(3-0.5 x_{i}\right) x_{i}+2 x_{i+1}-1, \\
i=2,3, \ldots, n-1, \\
F_{n}(x)=x_{n-1}-\left(3-0.5 x_{n}\right) x_{n}-1, \\
x_{0}=(-3,-3, \ldots,-3)^{T} .
\end{gathered}
$$

Problem 2 (Trigexp function [16]). The elements of $F(x)$ are

$$
\begin{gathered}
F_{1}(x)=3 x_{1}^{2}+2 x_{2}-5+\sin \left(x_{1}-x_{2}\right) \sin \left(x_{1}+x_{2}\right), \\
\begin{aligned}
F_{i}(x)=3 x_{i}^{2} & +2 x_{i+1}-5+\sin \left(x_{i}-x_{i+1}\right) \\
& \times \sin \left(x_{i}+x_{i+1}\right)+4 x_{i} \\
& -x_{i-1} \exp \left(x_{i}-1\right)-x_{i}-3 \\
i & =2,3, \ldots, n-1
\end{aligned} \\
F_{n}(x)=4 x_{n}-x_{n-1} \exp \left(x_{n-1}-x_{n}\right)-3 \\
x_{0}=(-2,-2, \ldots,-2)^{T} .
\end{gathered}
$$

Problem 3 (tridiagonal exponential problem [17]). The elements of $F(x)$ are

$$
\begin{aligned}
F_{1}(x) & =x_{1}-\exp \left(\cos \left(h\left(x_{1}+x_{2}\right)\right)\right), \\
F_{i}(x) & =x_{i}-\exp \left(\cos \left(h\left(x_{i-1}+x_{i}+x_{i+1}\right)\right)\right), \\
i=2,3, \ldots, n-1, & \\
F_{n}(x) & =x_{n}-\exp \left(\cos \left(h\left(x_{n-1}+x_{n}\right)\right)\right), \\
h & =\frac{1}{(n+1)}, \\
x_{0} & =(1.5,1.5, \ldots, 1.5)^{T} .
\end{aligned}
$$

Problem 4 (discrete boundary value problem [18]). The elements of $F(x)$ are

$$
\begin{aligned}
F_{1}(x) & =2 x_{1}+0.5 h^{2}\left(x_{1}+h\right)^{3}-x_{2}, \\
F_{i}(x) & =2 x_{i}+0.5 h^{2}\left(x_{i}+h i\right)^{3}-x_{i-1}+x_{i+1}, \\
i=2,3, \ldots, n-1, & \\
F_{n}(x) & =2 x_{n}+0.5 h^{2}\left(x_{n}+h n\right)^{3}-x_{n-1}, \\
h & =\frac{1}{n+1}, \\
x_{0} & =(h(h-1), h(2 h-1), \ldots, h(n h-1))^{T} .
\end{aligned}
$$

Problem 5 (exponentional problem 1 [19]). The elements of $F(x)$ are

$$
\begin{aligned}
F_{1}(x) & =\exp \left(x_{1}-1\right)-1, \\
F_{i}(x) & =i\left(\exp \left(x_{i}-1\right)-x_{i}\right), \quad i=2,3, \ldots, n, \\
x_{0} & =\left(\frac{n}{n-1}, \ldots, \frac{n}{n-1}\right)^{T} .
\end{aligned}
$$


TABLe 1: Results of Algorithm 3 for Problems 1-7.

\begin{tabular}{|c|c|c|c|c|c|c|c|c|}
\hline \multirow{2}{*}{ Pro } & \multirow{2}{*}{$n$} & \multirow{2}{*}{$\left\|F\left(x_{0}\right)\right\|$} & \multicolumn{3}{|c|}{$B_{0}=I$} & \multicolumn{3}{|c|}{$B_{0}=F^{\prime}\left(x_{0}\right)$} \\
\hline & & & $\left\|F\left(x_{k}\right)\right\|$ & Iter & Time (s) & $\left\|F\left(x_{k}\right)\right\|$ & Iter & Time (s) \\
\hline \multirow{9}{*}{1} & 50 & 26.8421 & $4.2686 e-06$ & 17 & 0.0000 & $3.0281 e-06$ & 12 & 0.0000 \\
\hline & 100 & 36.5103 & $6.6863 e-06$ & 17 & 0.0000 & $3.0284 e-06$ & 12 & 0.0000 \\
\hline & 200 & 50.5767 & $3.4126 e-06$ & 19 & 0.0313 & $3.0284 e-06$ & 12 & 0.0313 \\
\hline & 500 & 78.9494 & $3.5829 e-06$ & 19 & 0.0313 & $3.0284 e-06$ & 12 & 0.0313 \\
\hline & 1000 & 111.1665 & $6.7616 e-06$ & 20 & 0.0938 & $3.0284 e-06$ & 12 & 0.0625 \\
\hline & 3000 & 156.8247 & $4.6028 e-06$ & 21 & 0.1780 & $3.0284 e-06$ & 12 & 0.1563 \\
\hline & 5000 & 247.7055 & $5.0272 e-06$ & 22 & 0.2063 & $3.0284 e-06$ & 12 & 0.2031 \\
\hline & 10000 & 256.8910 & $5.6234 e-06$ & 25 & 0.2028 & $3.0284 e-06$ & 12 & 0.3125 \\
\hline & 20000 & 289.2351 & $5.4512 e-06$ & 25 & 0.3276 & $3.0284 e-06$ & 12 & 0.6563 \\
\hline \multirow{9}{*}{2} & 50 & 6.9282 & $1.3431 e-06$ & 10 & 0.0000 & $1.0235 e-06$ & 4 & 0.0000 \\
\hline & 100 & 9.8995 & $1.8437 e-06$ & 11 & 0.0000 & $1.0235 e-06$ & 4 & 0.0000 \\
\hline & 200 & 14.0712 & $6.2185 e-06$ & 11 & 0.0313 & $1.0235 e-06$ & 4 & 0.0000 \\
\hline & 500 & 22.3159 & $2.6805 e-06$ & 12 & 0.0313 & $1.0235 e-06$ & 4 & 0.0000 \\
\hline & 1000 & 31.5911 & $7.8148 e-06$ & 11 & 0.0625 & $1.0235 e-06$ & 4 & 0.0313 \\
\hline & 3000 & 54.7540 & $8.5561 e-06$ & 18 & 0.7188 & $1.0235 e-06$ & 4 & 0.0625 \\
\hline & 5000 & 70.6965 & $8.3005 e-06$ & 12 & 0.2500 & $1.0235 e-06$ & 4 & 0.0938 \\
\hline & 10000 & 75.0126 & $8.2145 e-06$ & 14 & 0.2808 & $1.0235 e-06$ & 4 & 0.1406 \\
\hline & 20000 & 81.2659 & $8.5179 e-06$ & 14 & 0.5304 & $1.0235 e-06$ & 4 & 0.2500 \\
\hline \multirow{9}{*}{3} & 50 & 8.5416 & $8.7650 e-08$ & 3 & 0.0000 & $1.1795 e-07$ & 5 & 0.0000 \\
\hline & 100 & 12.1562 & $6.8406 e-10$ & 3 & 0.0000 & $5.3904 e-06$ & 4 & 0.0000 \\
\hline & 200 & 17.2195 & $8.0105 e-06$ & 2 & 0.0000 & $3.6741 e-07$ & 3 & 0.0000 \\
\hline & 500 & 27.2392 & $3.3130 e-07$ & 2 & 0.0000 & $1.2337 e-06$ & 3 & 0.0000 \\
\hline & 1000 & 38.5246 & $2.9461 e-08$ & 2 & 0.0000 & $1.5498 e-07$ & 3 & 0.0313 \\
\hline & 3000 & 66.7279 & $6.3233 e-10$ & 3 & 0.0313 & $3.0014 e-06$ & 2 & 0.0313 \\
\hline & 5000 & 86.1455 & $1.0586 e-10$ & 2 & 0.0313 & $1.0804 e-06$ & 2 & 0.0625 \\
\hline & 10000 & 121.8282 & $9.3691 e-12$ & 2 & 0.0938 & $2.7008 e-07$ & 2 & 0.0938 \\
\hline & 20000 & 172.2911 & $8.7919 e-13$ & 2 & 0.1563 & $6.7517 e-08$ & 2 & 0.1719 \\
\hline \multirow{9}{*}{4} & 50 & 0.1511 & $8.7479 e-06$ & 19 & 0.0000 & $2.4065 e-06$ & 13 & 0.0000 \\
\hline & 100 & 0.1111 & $8.4206 e-06$ & 16 & 0.0000 & $5.7592 e-06$ & 12 & 0.0000 \\
\hline & 200 & 0.0801 & $9.4752 e-06$ & 18 & 0.0313 & $9.6492 e-06$ & 10 & 0.0313 \\
\hline & 500 & 0.0512 & $8.2454 e-06$ & 14 & 0.0313 & $9.0492 e-06$ & 10 & 0.0313 \\
\hline & 1000 & 0.0364 & $7.7380 e-06$ & 13 & 0.0625 & $4.0496 e-06$ & 10 & 0.0625 \\
\hline & 3000 & 0.0211 & $7.6951 e-06$ & 13 & 0.1719 & $9.7135 e-06$ & 8 & 0.1250 \\
\hline & 5000 & 0.0163 & $9.8163 e-06$ & 10 & 0.1875 & $8.4494 e-06$ & 7 & 0.1563 \\
\hline & 10000 & 0.0115 & $8.4212 e-06$ & 10 & 0.3125 & $4.1718 e-06$ & 7 & 0.2344 \\
\hline & 20000 & 0.0082 & $6.9072 e-06$ & 4 & 0.2656 & $2.0897 e-06$ & 7 & 0.3906 \\
\hline \multirow{9}{*}{5} & 50 & 0.0481 & $7.6492 e-06$ & 9 & 0.0000 & $5.5212 e-06$ & 9 & 0.0000 \\
\hline & 100 & 0.0315 & $5.3122 e-06$ & 9 & 0.0000 & $9.8754 e-06$ & 8 & 0.0000 \\
\hline & 200 & 0.0213 & $9.7441 e-06$ & 8 & 0.0000 & $6.8642 e-06$ & 8 & 0.0000 \\
\hline & 500 & 0.0131 & $6.1289 e-06$ & 8 & 0.0000 & $4.2970 e-06$ & 8 & 0.0000 \\
\hline & 1000 & 0.0092 & $4.3258 e-06$ & 8 & 0.0000 & $7.9238 e-06$ & 7 & 0.0000 \\
\hline & 3000 & 0.0053 & $6.5316 e-06$ & 7 & 0.0000 & $4.5644 e-06$ & 7 & 0.0000 \\
\hline & 5000 & 0.0041 & $5.0582 e-06$ & 7 & 0.0000 & $9.2636 e-06$ & 6 & 0.0000 \\
\hline & 10000 & 0.0029 & $9.3577 e-06$ & 6 & 0.0000 & $6.5482 e-06$ & 6 & 0.0313 \\
\hline & 20000 & 0.0020 & $6.6163 e-06$ & 6 & 0.0313 & $4.6295 e-06$ & 6 & 0.0313 \\
\hline
\end{tabular}


TABle 1: Continued.

\begin{tabular}{|c|c|c|c|c|c|c|c|c|}
\hline \multirow{2}{*}{ Pro } & \multirow{2}{*}{$n$} & \multirow{2}{*}{$\left\|F\left(x_{0}\right)\right\|$} & \multicolumn{3}{|c|}{$B_{0}=I$} & \multicolumn{3}{|c|}{$B_{0}=F^{\prime}\left(x_{0}\right)$} \\
\hline & & & $\left\|F\left(x_{k}\right)\right\|$ & Iter & Time (s) & $\left\|F\left(x_{k}\right)\right\|$ & Iter & Time (s) \\
\hline \multirow{9}{*}{6} & 50 & 0.0166 & $6.6166 e-06$ & 7 & 0.0000 & $6.5364 e-06$ & 5 & 0.0000 \\
\hline & 100 & 0.0116 & $7.5062 e-06$ & 6 & 0.0000 & $8.5483 e-06$ & 2 & 0.0000 \\
\hline & 200 & 0.0082 & $9.4436 e-06$ & 4 & 0.0000 & $2.1372 e-06$ & 2 & 0.0000 \\
\hline & 500 & 0.0052 & $9.8417 e-06$ & 18 & 0.0406 & $5.2204 e-06$ & 1 & 0.0000 \\
\hline & 1000 & 0.0037 & $9.6163 e-06$ & 24 & 0.0313 & $3.6586 e-06$ & 3 & 0.0313 \\
\hline & 3000 & 0.0021 & $8.3375 e-06$ & 17 & 0.0625 & $2.1088 e-06$ & 3 & 0.0313 \\
\hline & 5000 & 0.0016 & $9.7458 e-06$ & 15 & 0.0938 & $1.6333 e-06$ & 3 & 0.0313 \\
\hline & 10000 & 0.0012 & $9.9565 e-06$ & 11 & 0.1563 & $1.1548 e-06$ & 3 & 0.0313 \\
\hline & 20000 & 0.0082 & $8.8706 e-06$ & 9 & 0.2031 & $8.1653 e-06$ & 2 & 0.0625 \\
\hline \multirow{9}{*}{7} & 50 & 0.2271 & $1.4914 e-14$ & 2 & 0.0000 & $5.9494 e-06$ & 53 & 0.1250 \\
\hline & 100 & 0.2319 & $1.4792 e-14$ & 2 & 0.0000 & $5.6147 e-06$ & 58 & 0.0938 \\
\hline & 200 & 0.2413 & $1.4960 e-14$ & 2 & 0.0000 & $6.6486 e-06$ & 77 & 0.2031 \\
\hline & 500 & 0.2675 & $1.5760 e-14$ & 2 & 0.0000 & $7.4199 e-06$ & 70 & 0.7500 \\
\hline & 1000 & 0.3062 & 0 & 2 & 0.0313 & $8.2082 e-06$ & 35 & 0.3906 \\
\hline & 3000 & 0.4274 & 0 & 4 & 0.0625 & $5.7188 e-06$ & 53 & 10.5469 \\
\hline & 5000 & 0.5211 & 0 & 7 & 2.5000 & $6.7092 e-06$ & 41 & 19.0625 \\
\hline & 10000 & 0.7027 & 0 & 9 & 10.3125 & $6.8420 e-06$ & 45 & 25.0156 \\
\hline & 20000 & 0.9686 & 0 & 11 & 43.7500 & $7.1086 e-06$ & 51 & 30.0156 \\
\hline
\end{tabular}

Problem 6 (exponentional problem 2 [19]). The elements of $F(x)$ are

$$
\begin{aligned}
F_{1}(x) & =\exp \left(x_{1}\right)-1, \\
F_{i}(x) & =\frac{i}{10}\left(\exp \left(x_{i}\right)+x_{i-1}-1\right), \quad i=2,3, \ldots, n, \\
x_{0} & =\left(\frac{1}{n^{2}}, \ldots, \frac{1}{n^{2}}\right)^{T} .
\end{aligned}
$$

Problem 7 ( penalty I function [19]). The elements of $F(x)$ are

$$
\begin{aligned}
F_{i}(x) & =\sqrt{10^{-5}}\left(x_{i}-1\right), \quad i=1, \ldots, n-1, \\
F_{n}(x) & =\frac{1}{4 n} \sum_{j=1}^{n} x_{j}^{2}-\frac{1}{4}, \\
x_{0} & =\left(\frac{1}{3}, \ldots, \frac{1}{3}\right)^{T} .
\end{aligned}
$$

Problem 8 (exponential function [19]). The elements of $F(x)$ are

$$
\begin{aligned}
F_{i}(x) & =\frac{i}{10}\left(1-x_{i}^{2}-\exp \left(-x_{i}^{2}\right)\right), \quad i=1,2, \ldots, n-1, \\
F_{n}(x) & =\frac{n}{10}\left(1-\exp \left(-x_{n}^{2}\right)\right), \\
x_{0} & =\left(\frac{1}{4 n^{2}}, \frac{2}{4 n^{2}}, \ldots, \frac{n}{4 n^{2}}\right)^{T} .
\end{aligned}
$$

Problem 9 (minimal function [19]). The elements of $F(x)$ are

$$
\begin{aligned}
F_{i}(x) & =\frac{\left(\ln x_{i}-\exp \left(x_{i}\right)\right)-\sqrt{\left(\ln x_{i}-\exp \left(x_{i}\right)\right)^{2}+10^{-10}}}{2}, \\
x_{0} & =(1,1, \ldots, 1)^{T} .
\end{aligned}
$$

Problem 10 (extended Rosenbrock function ( $n$ is even) [20]). The elements of $F(x)$ are

$$
\begin{aligned}
F_{2 i-1}(x) & =10\left(x_{2 i}-x_{2 i-1}^{2}\right), \\
F_{2 i}(x) & =1-x_{2 i-1}, \quad i=1,2, \ldots, \frac{n}{2}, \\
x_{0} & =(5,1,5,1, \ldots, 5,1)^{T} .
\end{aligned}
$$

Problem 11 (logarithmic function [19]). The elements of $F(x)$ are

$$
\begin{aligned}
F_{i}(x) & =\ln \left(x_{i}+1\right)-\frac{x_{i}}{n}, \quad i=1,2, \ldots, n, \\
x_{0} & =(1,1, \ldots, 1)^{T} .
\end{aligned}
$$

Problem 12 (strictly convex function 1 [21]). $F(x)$ is the gradient of $f(x)=\sum_{i=1}^{n}\left(\exp \left(x_{1}\right)-x_{i}\right)$. The elements of $F(x)$ are

$$
\begin{aligned}
F_{i}(x) & =\exp \left(x_{i}\right)-1, \quad i=1,2, \ldots, n . \\
x_{0} & =\left(\frac{1}{n}, \frac{2}{n}, \ldots, 1\right)^{T} .
\end{aligned}
$$


TABLe 2: Results of Algorithm 3 for Problems 8-14.

\begin{tabular}{|c|c|c|c|c|c|c|c|c|}
\hline \multirow{2}{*}{ Pro } & \multirow{2}{*}{$n$} & \multirow{2}{*}{$\left\|F\left(x_{0}\right)\right\|$} & \multicolumn{3}{|c|}{$B_{0}=I$} & \multicolumn{3}{|c|}{$B_{0}=F^{\prime}\left(x_{0}\right)$} \\
\hline & & & $\left\|F\left(x_{k}\right)\right\|$ & Iter & Time (s) & $\left\|F\left(x_{k}\right)\right\|$ & Iter & Time $(\mathrm{s})$ \\
\hline \multirow{9}{*}{8} & 52 & $1.2019 e-04$ & $4.7426 e-06$ & 4 & 0.0000 & $4.8145 e-06$ & 6 & 0.0000 \\
\hline & 100 & $6.2500 e-05$ & $6.8402 e-06$ & 3 & 0.0000 & $6.9537 e-06$ & 4 & 0.0000 \\
\hline & 200 & $3.1250 e-05$ & $7.7243 e-06$ & 2 & 0.0000 & $7.8200 e-06$ & 2 & 0.0000 \\
\hline & 500 & $1.2500 e-05$ & $9.8751 e-06$ & 11 & 0.0000 & $3.1279 e-06$ & 2 & 0.0000 \\
\hline & 1000 & $6.2500 e-06$ & $6.2500 e-06$ & 0 & 0.0000 & $6.2500 e-06$ & 0 & 0.0000 \\
\hline & 3000 & $2.0833 e-06$ & $2.0833 e-06$ & 0 & 0.0000 & $2.0833 e-06$ & 0 & 0.0000 \\
\hline & 5000 & $1.2500 e-06$ & $1.2500 e-06$ & 0 & 0.0000 & $1.2500 e-06$ & 0 & 0.0000 \\
\hline & 10000 & $6.2500 e-07$ & $6.2500 e-07$ & 0 & 0.0000 & $6.2500 e-07$ & 0 & 0.0000 \\
\hline & 20000 & $3.1250 e-07$ & $3.1250 e-07$ & 0 & 0.0000 & $3.1250 e-07$ & 0 & 0.0000 \\
\hline \multirow{9}{*}{9} & 50 & 19.2212 & $4.7930 e-11$ & 4 & 0.0000 & $4.3678 e-11$ & 2 & 0.0000 \\
\hline & 100 & 27.1828 & $6.7783 e-11$ & 4 & 0.0000 & $6.1770 e-11$ & 2 & 0.0000 \\
\hline & 200 & 38.4423 & $9.5860 e-11$ & 4 & 0.0000 & $9.8689 e-11$ & 2 & 0.0000 \\
\hline & 500 & 60.7826 & $1.5157 e-10$ & 4 & 0.0000 & $1.5604 e-10$ & 2 & 0.0000 \\
\hline & 1000 & 85.9596 & $2.1435 e-10$ & 4 & 0.0313 & $2.2068 e-10$ & 2 & 0.0000 \\
\hline & 3000 & 148.8864 & $4.1536 e-10$ & 4 & 0.0313 & $3.8222 e-10$ & 2 & 0.0313 \\
\hline & 5000 & 192.2116 & $5.3623 e-10$ & 4 & 0.0625 & $4.9345 e-10$ & 2 & 0.0625 \\
\hline & 10000 & 271.8282 & $7.5834 e-10$ & 4 & 0.1250 & $7.7298 e-10$ & 2 & 0.0938 \\
\hline & 20000 & 384.4231 & $1.0725 e-09$ & 4 & 0.2500 & $1.0932 e-09$ & 2 & 0.1563 \\
\hline \multirow{9}{*}{10} & 50 & 1200 & $8.3426 e-06$ & 33 & 0.0000 & $4.2841 e-09$ & 19 & 0.0000 \\
\hline & 100 & 1697.1 & $2.2053 e-07$ & 34 & 0.0000 & $6.0586 e-09$ & 19 & 0.0000 \\
\hline & 200 & 2400 & $2.4538 e-07$ & 34 & 0.0000 & $3.5281 e-06$ & 23 & 0.0000 \\
\hline & 500 & 3794.7 & $1.3524 e-06$ & 35 & 0.0313 & $5.5783 e-06$ & 23 & 0.0000 \\
\hline & 1000 & 5366.6 & $1.0785 e-06$ & 33 & 0.0313 & $7.8890 e-06$ & 23 & 0.0000 \\
\hline & 3000 & 9295.2 & $2.3859 e-07$ & 33 & 0.0625 & $1.1640 e-07$ & 24 & 0.0313 \\
\hline & 5000 & 12000 & $3.0801 e-07$ & 33 & 0.0938 & $1.5028 e-07$ & 24 & 0.0625 \\
\hline & 10000 & 16971 & $4.1301 e-06$ & 29 & 0.1563 & $2.1252 e-07$ & 24 & 0.1250 \\
\hline & 20000 & 24000 & $5.3904 e-06$ & 25 & 0.2813 & $1.4378 e-07$ & 24 & 0.2500 \\
\hline \multirow{9}{*}{11} & 50 & 4.7599 & $1.2153 e-07$ & 6 & 0.0000 & $1.4006 e-06$ & 6 & 0.0000 \\
\hline & 100 & 6.8315 & $1.1647 e-07$ & 6 & 0.0000 & $1.4743 e-06$ & 6 & 0.0000 \\
\hline & 200 & 9.7319 & $1.3534 e-07$ & 6 & 0.0000 & $1.8019 e-06$ & 6 & 0.0000 \\
\hline & 500 & 15.4545 & $1.9008 e-07$ & 6 & 0.0000 & $2.6117 e-06$ & 6 & 0.0000 \\
\hline & 1000 & 21.8876 & $2.5837 e-07$ & 6 & 0.0000 & $3.5883 e-06$ & 6 & 0.0000 \\
\hline & 3000 & 37.9470 & $4.3584 e-07$ & 6 & 0.0000 & $6.0967 e-06$ & 6 & 0.0000 \\
\hline & 5000 & 48.9988 & $5.5970 e-07$ & 6 & 0.0000 & $2.6853 e-06$ & 6 & 0.0000 \\
\hline & 10000 & 69.3047 & $7.8840 e-07$ & 6 & 0.0313 & $3.7947 e-06$ & 6 & 0.0313 \\
\hline & 20000 & 98.0187 & $1.1128 e-06$ & 6 & 0.0313 & $5.3645 e-06$ & 6 & 0.0469 \\
\hline \multirow{9}{*}{12} & 50 & 6.2761 & $1.5871 e-08$ & 7 & 0.0000 & $2.7576 e-08$ & 6 & 0.0000 \\
\hline & 100 & 8.7909 & $1.9036 e-08$ & 7 & 0.0000 & $3.6023 e-08$ & 6 & 0.0000 \\
\hline & 200 & 12.3723 & $2.4543 e-08$ & 7 & 0.0000 & $4.8866 e-08$ & 6 & 0.0000 \\
\hline & 500 & 19.5054 & $3.6586 e-08$ & 7 & 0.0000 & $7.5309 e-08$ & 6 & 0.0000 \\
\hline & 1000 & 27.5580 & $5.0705 e-08$ & 7 & 0.0000 & $1.0559 e-07$ & 6 & 0.0000 \\
\hline & 3000 & 47.7008 & $8.6635 e-08$ & 7 & 0.0000 & $1.8182 e-07$ & 6 & 0.0000 \\
\hline & 5000 & 61.5735 & $1.1154 e-07$ & 7 & 0.0000 & $2.3446 e-07$ & 6 & 0.0000 \\
\hline & 10000 & 87.0696 & $1.5742 e-07$ & 7 & 0.0000 & $3.3129 e-07$ & 6 & 0.0000 \\
\hline & 20000 & 123.1291 & $2.2239 e-07$ & 7 & 0.0313 & $4.6831 e-07$ & 6 & 0.0313 \\
\hline
\end{tabular}


TABLe 2: Continued.

\begin{tabular}{|c|c|c|c|c|c|c|c|c|}
\hline \multirow[t]{2}{*}{ Pro } & \multirow[t]{2}{*}{$n$} & \multirow{2}{*}{$\left\|F\left(x_{0}\right)\right\|$} & \multicolumn{3}{|c|}{$B_{0}=I$} & \multicolumn{3}{|c|}{$B_{0}=F^{\prime}\left(x_{0}\right)$} \\
\hline & & & $\left\|F\left(x_{k}\right)\right\|$ & Iter & Time (s) & $\left\|F\left(x_{k}\right)\right\|$ & Iter & Time (s) \\
\hline \multirow{9}{*}{13} & 51 & 771.2349 & $8.4607 e-09$ & 10 & 0.0000 & $1.6413 e-08$ & 9 & 0.0000 \\
\hline & 99 & 1089.8 & $1.1644 e-08$ & 10 & 0.0000 & $2.3210 e-08$ & 9 & 0.0000 \\
\hline & 201 & 1564.5 & $1.6479 e-08$ & 10 & 0.0000 & $3.3331 e-08$ & 9 & 0.0000 \\
\hline & 501 & 2480.5 & $2.5914 e-08$ & 10 & 0.0000 & $5.2860 e-08$ & 9 & 0.0000 \\
\hline & 999 & 3507.7 & $3.6544 e-08$ & 10 & 0.0000 & $7.4754 e-08$ & 9 & 0.0000 \\
\hline & 3000 & 6087.3 & $6.3242 e-08$ & 10 & 0.0313 & $1.2974 e-07$ & 9 & 0.0000 \\
\hline & 5001 & 7857.2 & $8.1675 e-08$ & 10 & 0.0625 & $1.6746 e-07$ & 9 & 0.0313 \\
\hline & 9999 & 11112 & $1.1547 e-07$ & 10 & 0.0938 & $2.3682 e-07$ & 9 & 0.0625 \\
\hline & 20001 & 15717 & $1.6330 e-07$ & 10 & 0.1563 & $3.3496 e-07$ & 9 & 0.1563 \\
\hline \multirow{9}{*}{14} & 50 & 147.1394 & $2.8039 e-06$ & 7 & 0.0000 & $1.4943 e-06$ & 8 & 0.0000 \\
\hline & 100 & 208.0865 & $3.9654 e-06$ & 7 & 0.0000 & $2.1133 e-06$ & 8 & 0.0000 \\
\hline & 200 & 294.2788 & $5.6079 e-06$ & 7 & 0.0000 & $2.9887 e-06$ & 8 & 0.0313 \\
\hline & 500 & 465.2956 & $8.8669 e-06$ & 7 & 0.0313 & $4.7255 e-06$ & 8 & 0.0625 \\
\hline & 1000 & 658.0274 & $3.5104 e-07$ & 8 & 0.0625 & $6.6829 e-06$ & 8 & 0.1250 \\
\hline & 3000 & 1139.7 & $6.0803 e-07$ & 8 & 0.1250 & $3.1348 e-08$ & 9 & 0.3438 \\
\hline & 5000 & 1471.4 & $7.8496 e-07$ & 8 & 0.2969 & $4.0470 e-08$ & 9 & 0.4219 \\
\hline & 10000 & 2080.9 & $1.1101 e-06$ & 8 & 0.3594 & $5.7234 e-08$ & 9 & 0.8438 \\
\hline & 20000 & 2942.8 & $1.5699 e-06$ & 8 & 0.7656 & $8.0941 e-08$ & 9 & 1.5000 \\
\hline
\end{tabular}

Problem 13 (tridimensional valley function ( $n$ is a multiple of 3) [22]). The elements of $F(x)$ are

$$
\begin{aligned}
F_{3 i-2}(x) & =\left(c_{2} x_{3 i-2}^{3}+c_{1} x_{3 i-2}\right) \exp \left(\frac{-x_{3 i-2}^{2}}{100}\right)-1, \\
F_{3 i-1}(x) & =10\left(\sin \left(x_{3 i-2}-x_{3 i-1}\right)\right), \\
F_{3 i}(x) & =10\left(\cos \left(x_{3 i-2}-x_{3 i}\right)\right), \quad i=1,2, \ldots, \frac{n}{3},
\end{aligned}
$$

and then denote

$$
\begin{aligned}
& c_{1}=1.003344481605351, \\
& c_{2}=-3.344481605351171 \times 10^{-3}, \\
& x_{0}=(2,1,2,1,2, \ldots, 1)^{T} .
\end{aligned}
$$

Problem 14 (extended Freudenstein and Roth function ( $n$ is even) [17]). The elements of $F(x)$ are

$$
\begin{gathered}
F_{2 i-1}(x)=x_{2 i-1}+\left(\left(5-x_{2 i}\right) x_{2 i}-2\right) x_{2 i}-13, \\
F_{2 i}(x)=x_{2 i-1}+\left(\left(x_{2 i}+1\right) x_{2 i}-14\right) x_{2 i}-29, \\
i=1,2, \ldots, \frac{n}{2}, \\
x_{0}=(6,3,6,3, \ldots, 6,3)^{T} .
\end{gathered}
$$

The sparsity patterns of most of the problems are tridiagonal and the dimension of problems varies from 50 to 20000 . The results are given in Tables 1 and 2, and each column is specified as follows:
Pro: the problem;

$n$ : the dimension of the problem;

$\left\|F\left(x_{0}\right)\right\|:$ the initial Euclidean norms of $F(x)$;

$\left\|F\left(x_{k}\right)\right\|:$ the final Euclidean norms of $F(x)$;

Iter: the total number of iterations;

Times: the CPU time in second.

It can be seen from the tables that, for all tested problems, Algorithm 3 terminated successfully. The numerical results show that Algorithm 3 becomes increasingly desirable as $n$ increases.

In Tables 1 and 2, we list the results of Algorithm 3 for solving Problems 1 to 14 with $B_{0}=I$. Because $B_{0}$ is very important for the performance of Broyden's method, we also present the results with $B_{0}=F^{\prime}\left(x_{0}\right)$. We can see that Algorithm 3 can be applied to solve a class of nonlinear equations, where the dimension of which can be up to 20000 . Since the Schubert's update formula (7) can maintain the sparsity pattern of Jacobian matrix exactly, so Algorithm 3 is especially effective for solving large-scale nonlinear equations with sparse Jacobian matrix, such as tridiagonal or block diagonal Jacobian matrix.

\section{Remarks}

In this paper, based on the work of Schubert, Broyden, and Marwil, we have globalized Schubert's method and proposed a global algorithm by using a nonmonotone line search. We have established the global and superlinear convergence. Numerical results showed that the algorithm is especially effective for large-scale problems. 


\section{Conflict of Interests}

The author declares that there is no conflict of interests regarding to the publication of this paper.

\section{Acknowledgment}

The work is supported by the National Science Foundation of China through Grant 11371154.

\section{References}

[1] Y. Yuan, "Recent advances in numerical methods for nonlinear equations and nonlinear least squares," Numerical Algebra, Control and Optimization, vol. 1, no. 1, pp. 15-34, 2011.

[2] L. K. Schubert, "Modification of a quasi-Newton method for nonlinear equations with a sparse Jacobian," Mathematics of Computation, vol. 24, pp. 27-30, 1970.

[3] E. Marwil, "Convergence results for Schubert's method for solving sparse nonlinear equations," SIAM Journal on Numerical Analysis, vol. 16, no. 4, pp. 588-604, 1979.

[4] C. G. Broyden, "The convergence of an algorithm for solving sparse nonlinear systems," Mathematics of Computation, vol. 25, no. 114 , pp. 285-294, 1971.

[5] B. Lam, "On the convergence of a quasi-Newton method for sparse nonlinear systems," Mathematics of Computation, vol. 32, no. 142, pp. 447-451, 1978.

[6] P. L. Toint, "On sparse and symmetric matrix updating subject to a linear equation," Mathematics of Computation, vol. 31, no. no 140, pp. 954-961, 1977.

[7] D. F. Shanno, "On variable-metric methods for sparse Hessians," Mathematics of Computation, vol. 34, no. 150, pp. 499-514, 1980.

[8] R. Fletcher, "An optimal positive definite update for sparse Hessian matrices," SIAM Journal on Optimization, vol. 5, no. 1, pp. 192-218, 1995.

[9] N. Yamashita, "Sparse quasi-Newton updates with positive definite matrix completion," Mathematical Programming, vol. 115, no. 1, pp. 1-30, 2008.

[10] Y. H. Dai and N. Yamashita, "Analysis of sparse quasi-Newton updates with positive definite matrix completion," Journal of the Operations Research Society of China, vol. 2, no. 1, pp. 39-56, 2014.

[11] P. L. Toint, "Global convergence of the partitioned BFGS algorithm for convex partially separable optimization," Mathematical Programming, vol. 36, no. 3, pp. 290-306, 1986.

[12] A. Griewank, "The global convergence of partitioned BFGS on problems with convex decompositions and Lipschitzian gradients," Mathematical Programming, vol. 50, no. 1-3, pp. 141-175, 1991.

[13] D. Li and M. Fukushima, "A derivative-free line search and global convergence of Broyden-like method for nonlinear equations," Optimization Methods and Software, vol. 13, no. 3, pp. 181-201, 2000.

[14] A. Griewank, "The "global" convergence of Broyden-like methods with a suitable line search," Australian Mathematical Society. Journal. Series B. Applied Mathematics, vol. 28, no. 1, pp. 75-92, 1986.

[15] C. G. Broyden, "A class of methods for solving nonlinear simultaneous equations," Mathematics of Computation, vol. 19, no. 92, pp. 577-593, 1965.
[16] M. A. Gomes-Ruggiero, J. M. Martínez, and A. C. Moretti, "Comparing algorithms for solving sparse nonlinear systems of equations," SIAM Journal on Scientific and Statistical Computing, vol. 13, no. 2, pp. 459-483, 1992.

[17] B. Yang and L. Gao, "An efficient implementation of Merrill's method for sparse or partially separable systems of nonlinear equations," SIAM Journal on Optimization, vol. 1, no. 2, pp. 206221, 1991.

[18] J. J. Moré, B. S. Garbow, and K. E. Hillström, “Testing unconstrained optimization software," ACM Transactions on Mathematical Software (TOMS), vol. 7, no. 1, pp. 17-41, 1981.

[19] W. La Cruz, J. M. Martnez, and M. Raydan, "Spectral residual method without gradient information for solving large-scale nonlinear systems of equations," Mathematics of Computation, vol. 75, no. 255, pp. 1429-1448, 2006.

[20] M. G. Gasparo, "A nonmonotone hybrid method for nonlinear systems," Optimization Methods and Software, vol. 13, no. 2, pp. 79-94, 2000.

[21] M. Raydan, "The Barzilai and Borwein gradient method for the large scale unconstrained minimization problem," SIAM Journal on Optimization, vol. 7, no. 1, pp. 26-33, 1997.

[22] A. Friedlander, M. A. Gomes-Ruggiero, D. N. Kozakevich, J. M. Martínez, and S. A. Santosa, "Solving nonlinear systems of equations by means of quasi-newton methods with a nonmonotone strategy," Optimization Methods and Software, vol. 8, no. 1, pp. 25-51, 1997. 


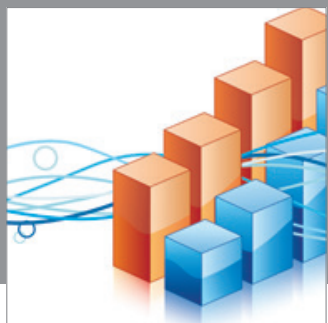

Advances in

Operations Research

mansans

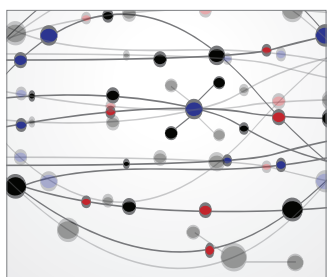

The Scientific World Journal
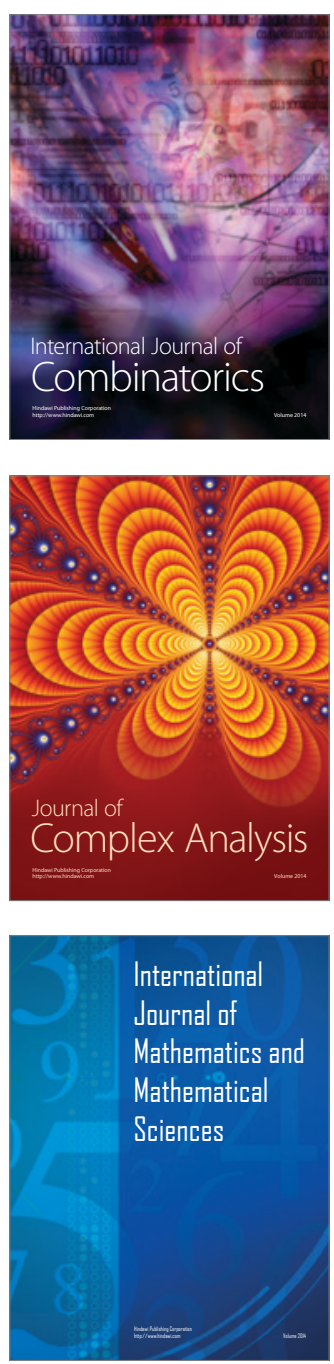
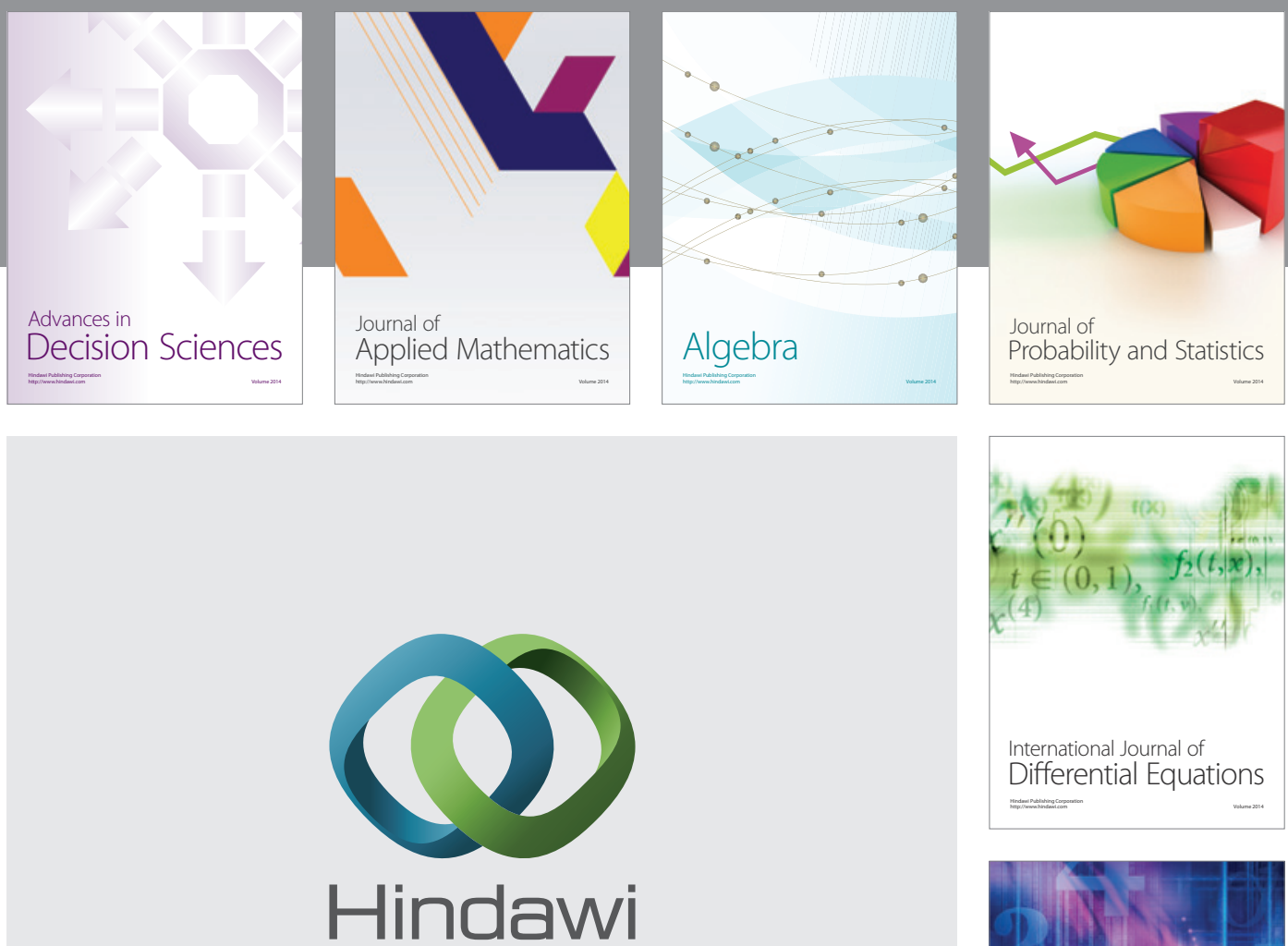

Submit your manuscripts at http://www.hindawi.com
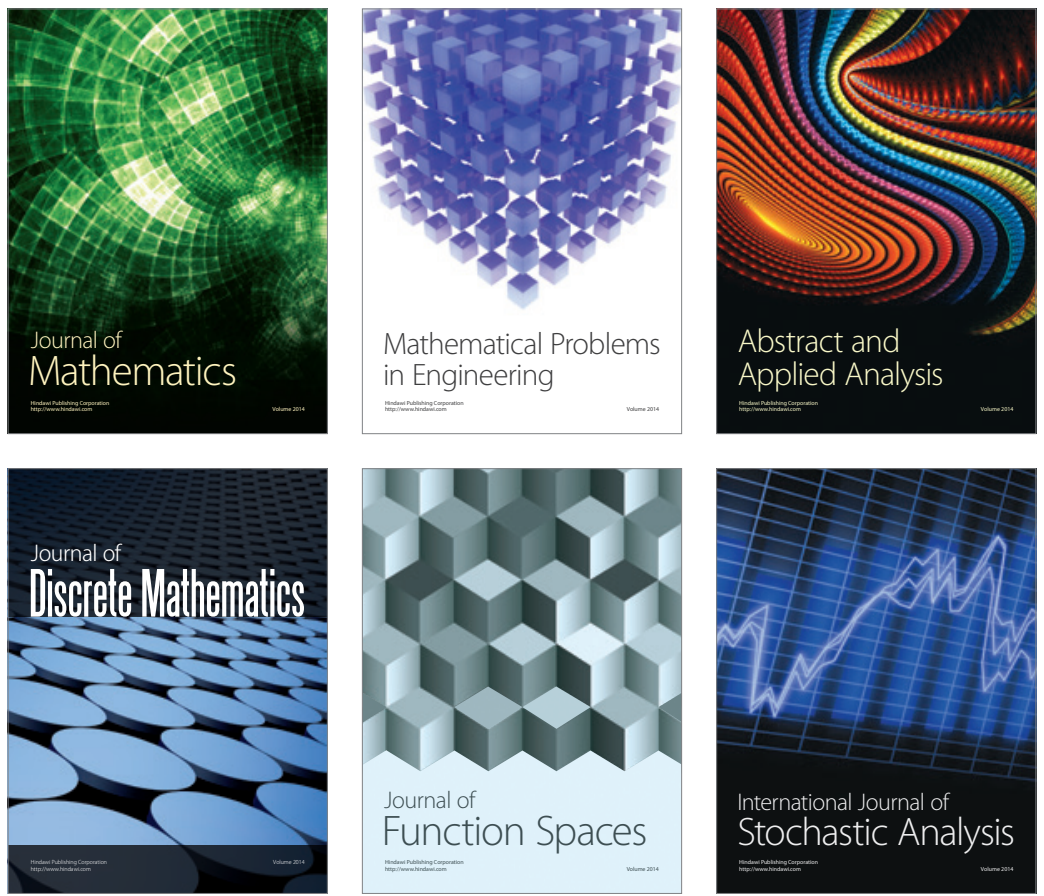

Journal of

Function Spaces

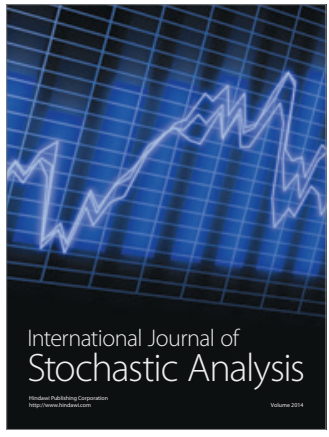

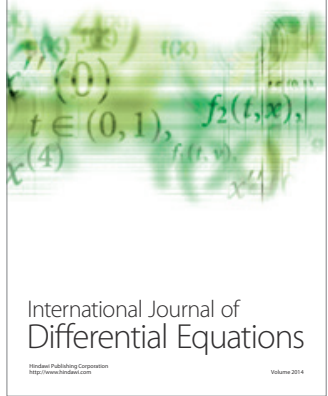
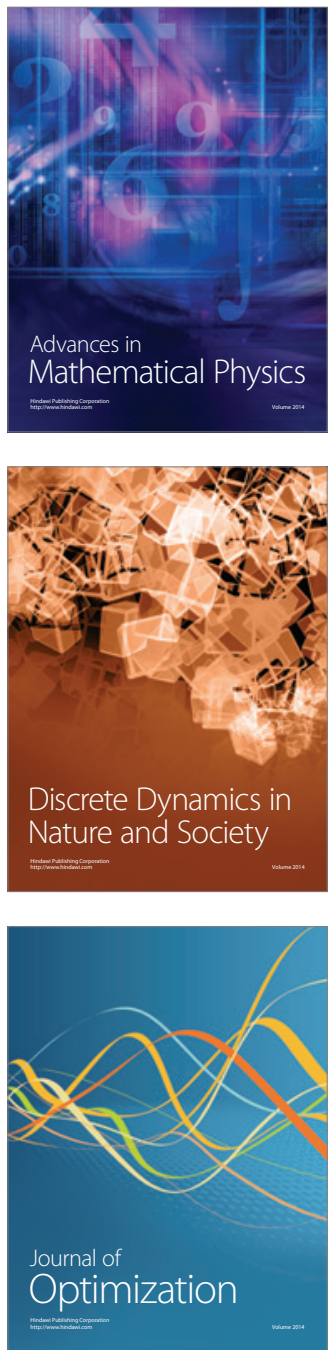